\title{
A New Design Algorithm for Two-Band Orthonormal Rational Filter Banks and Orthonormal Rational Wavelets
}

\author{
Thierry Blu
}

\begin{abstract}
In this paper, we present a new algorithm for the design of orthonormal two-band rational filter banks. Owing to the connection between iterated rational filter banks and rational wavelets, this is also a design algorithm for orthonormal rational wavelets. It is basically a simple iterative procedure, which explains its exponential convergence and adaptability under various linear constraints (e.g., regularity). Although the filters obtained from this algorithm are suboptimally designed, they show excellent frequency selectivity.

After an in-depth account of the algorithm, we discuss the properties of the rational wavelets generated by some designed filters. In particular, we stress the possibility to design "almost" shift error-free wavelets, which allows the implementation of a rational wavelet transform.
\end{abstract}

\section{INTRODUCTION}

$\mathbf{F}$ OR MOST signals, the time and frequency content have to be analyzed together since they convey complementary information, and this is usually done by using a battery of filters, i.e., a filter bank. However, due to the time-frequency uncertainty relations, a subtle tradeoff has to be found between the time and frequency resolution of the analysis filters: These the basics of filter design. Actually, it is often assumed that the filters have a finite impulse response (FIR) since this ensures that they are well localized in time, have finite delay, and require simple hardware implementation.

Many efficient filter design tools are available, and they provide different solutions depending on the choice of measure of the time-frequency resolution. However, for source coding applications, it is necessary to set new constraints: nonredundancy of the information, which results in subsampling, and stability of the reconstruction, which ensures that the coding-decoding scheme is not sensitive to quantization within the subbands. Critically sampled quadrature mirror filter (QMF) banks [1], [2] were then introduced in order to take these additional constraints into account: Although reconstruction is not perfect, aliasing is exactly cancelled so that the whole analysis-synthesis system behaves approximately as an allpass filter.

Manuscript received August 23, 1996; revised November 6, 1997. The associate editor coordinating the review of this paper and approving it for publication was Dr. Jelena Kovačević.

The author is with the Swiss Federal Institute of Technology, Lausanne, Switzerland, on leave from France Télécom CNET DSE/SGV, Issy-lesMoulineaux, France (e-mail: thierry.blu@cnet.francetelecom.fr)

Publisher Item Identifier S 1053-587X(98)03912-9.
Conjugate quadrature filters (CQF's) [3], [4] made it clear that it was possible to have both FIR and perfect reconstruction. The research in this field [5] was then boosted, resulting in an algebraic description of a filter bank by a polyphase matrix, the determinant of which was shown to characterize perfect reconstruction with FIR filters. An essential achievement of this formulation was the mathematical expression of the CQF property known as "paraunitarity," which resulted in the proof [6] that all CQF filter banks can be (robustly) implemented by cascading simple paraunitary elements of degree 1 . Not only does this factorization provide new implementation solutions, it is actually a powerful tool for the design of orthonormal filter banks [7], even though the method relies on a strongly nonlinear minimization algorithm. In the biorthogonal case [8], [9], other fruitful, though incomplete, factorization results have been devised as well.

At that time, the stress was put on uniform filter banks, whereas in many cases (e.g., signal with nonuniform spectrum), nonuniform structures could arguably prove more efficient. The link between wavelets and iterated (dyadic) twoband filter banks [10], [11] then generated a sudden interest in nonuniform filter banks, especially in the orthonormal case for which quite a few design algorithms [4], [12]-[14] were presented. The more involved biorthogonal case can be approached by using general (but expensive) design methods [9], [15].

The rational extension of the iterated dyadic filter banks was first proposed by Kovačević and Vetterli [16]. The reason for this extension is that noninteger scale factor analysis brings the advantage of a finer frequency resolution and is known, for example, to be more accurately tuned to the human auditory analysis system. A theoretical study [17], [18] showed that an iterated rational filter bank can be viewed approximately as a wavelet transform; more specifically, an intrinsic shift variance prevents this relation from being exact, although clues suggest that the generating filter can be designed to minimize the resulting shift error [34]. Unfortunately, this hypothesis could hardly be tested in the specific two-band orthonormal rational case since no design algorithm existed. The general methods [7], [15] are so heavy and CPU power consuming that they cannot practically qualify for intensive design procedures; other approximate methods [19], [20] do not qualify either since they do not ensure perfect reconstruction. 


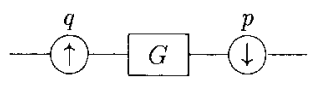

Fig. 1. Rational branch.

This led us to devise a simple, yet efficient design algorithm [21], especially dedicated to the rational case. In contrast with other methods that try to minimize the attenuation together with the reconstruction error, ours focuses only on the attenuation and relies on iterations to decrease the reconstruction error; an advantage of this approach is that the levels of these two errors are not linked together, as is usually the case (e.g., [19]). Furthermore, perfect reconstruction is mathematically ensured when the algorithm terminates (i.e., when the iterations converge); it provides exactly orthonormal filters that generate orthonormal rational wavelets.

This algorithm, which was first presented in [21], will be explained in detail after an introduction on rational filter banks and will then be illustrated with design examples.

\section{A. Notations}

All the filters are assumed to be FIR. We denote the $z$ transform of a filter $G(z)$ by $G(z)=\sum_{n} g[n] z^{-n}$. The filter is written in upper case, and its coefficients are written in lower case.

The $k$ th $p$-polyphase version of this filter is denoted by $G_{k}^{p}(z)$, where

$$
G_{k}^{p}(z)=\sum_{n} g_{k}^{p} z^{-n}=\sum_{n} g[k+n p] z^{-n} .
$$

The complex conjugate of any object $x$ is written $\bar{x}$, and the notation $\bar{G}(z)$ refers to a filter whose coefficients are conjugates of the coefficients of $G(z)$.

The $L$ th root of the unity $\zeta_{L}=e^{\frac{2 i \pi}{L}}$ is used in some equations.

Vectors are written in roman fonts, whereas matrices are in bold roman. This convention helps distinguish between a filter $G(z)$, a vector $G$, or a polynomial vector $G(z)$, and a matrix $\mathbf{G}$ or a polynomial matrix $\mathbf{G}(z)$.

The identity matrix is designated $\mathbf{I}$. The dimension of this matrix remains implicit since no ambiguity will arise from our equations.

\section{RATiONAL Filter BANKS (RFB)}

This section gives an introduction to rational filter banks, putting the emphasis on two-band structures and their iterations [16], [17], [22].

RFB's are made of elementary branches such as in Fig. 1. They can be either of the analysis kind (one input-multiple outputs) or of the synthesis kind (multiple inputs-one output), as shown in Fig. 2. The conservation of the sampling rate through the analysis (or the synthesis) stage, which is termed critical sampling, is mathematically enforced by the constraint $\sum_{i=0}^{N-1} \frac{q_{i}}{p_{i}}=1$. In the two-band case, the inverse structure of an "analysis RFB" is always a "synthesis RFB," although this is not true in the general $N$-band case [16], [23].

A tree structure is a simple way to build complex filter banks out of simpler ones, and this property carries over to

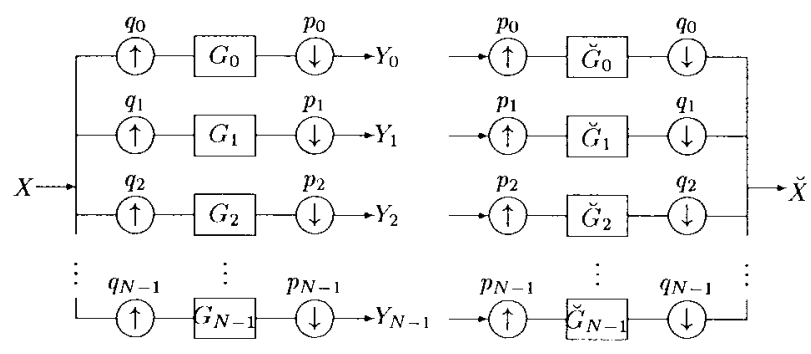

Fig. 2. Analysis (left) and synthesis (right) rational filter banks.

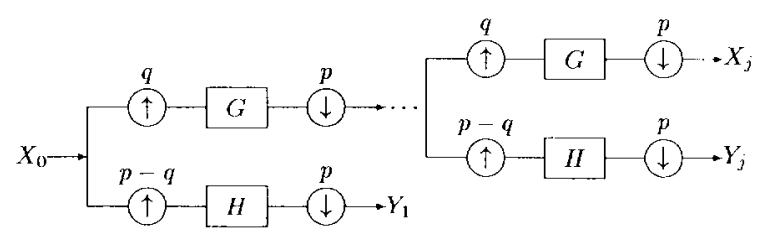

Fig. 3. Iterated two-band rational filter bank and limit functions.

RFB's generated by the iteration of two-band filter banks. In this paper, we consider only iterations over the same branch (the lowpass one) such as in Fig. 3 and show how to design the involved filters so that the resulting RFB preserves "good" properties.

Structurally, a rational branch (Fig. 1) performs a fractional sampling rate change. In the frequency domain, this property extends the usual halfband filtering behavior that characterizes the dyadic case (i.e., the case $p=2$ and $q=1$ ) by allowing the bandwidth to be any fraction $(<1)$ of the sampling interval. This makes it possible to implement constant $\Delta f / f$ transforms through iterations (as depicted in Fig. 3) with almost no restriction on the scale factor (it only has to be a rational number). This freedom is a significant improvement over the dyadic case where the scale factor is frozen at 2.

This finds a natural application in audio sound processing, for which an octave-band analysis is ill adapted. Instead, an iterated rational filter bank with a scale factor $p / q=6 / 5$ [24] closely approximates the Bark scale analysis [33] and, thus, makes it structurally easy to take psychoacoustic masking effects into account.

\section{A. Two-Band Iterated Rational Filter Banks (IRFB's)}

We have proved in an earlier paper [17] that iterating a rational filter bank on its lowpass branch yields limit functions in a very similar way as in the dyadic case. The difference is that these functions are not shift invariant when the generating lowpass filter $G(z)$ has a finite impulse response [16], [25], and thus, they cannot rigorously be termed "wavelets." They are instead named "pseudo-wavelets" [17]. For the sake of simplicity, however, we shall not retain this terminology and call them "rational wavelets," owing to the many properties they share with true wavelets [18].

As a matter of fact, the decomposition of a signal by an IRFB can be interpreted within the multiresolution analysis formalism [10], [11] (i.e., embedded multiresolution functional spaces $\ldots V_{-1} \subset V_{0} \ldots V_{n} \ldots$ and associated complementary biorthogonal spaces $W_{n}$ with $\left.V_{n} \oplus W_{n}=V_{n+1}\right)$. As pointed out earlier, the spaces $V_{n}$ are generated by a set of nonshifted 
functions $\left\{\varphi_{j}\left(\frac{p^{n}}{q^{n}} t\right)\right\}_{j \in \mathbb{Z}}$ (instead of $\left\{\varphi\left(2^{n} t-j\right)\right\}_{j \in \mathbb{Z}}$ in the dyadic case).

Many results carry over, with adequate adaptation, from the dyadic to the rational case. In particular, it is still possible to compute the global regularity of the limit functions (here, the minimum value of the Hölder exponents of the functions) via an infinite product of matrices [26], [27, dyadic case], [28, rational case]. Actually, the rational fraction $R(z)=$ $\frac{q}{p} z^{-p}-1$ plays the role of a regularity factor, but unlike its integer equivalent $1+z^{-1}+z^{-2}+\cdots z^{-p+1}$, this is never a polynomial when $q \geq 2$. However, requiring that $G(z)$ contains $N$ regularity factors and that the true polynomial $R_{0}(z)^{N}=\left(\frac{z^{-p}-1}{z^{-1}-1}\right)^{N}$ divides $G(z)$ are equivalent conditions for FIR filters.

\section{B. Orthonormal IRFB's}

We shall now focus on orthonormal filter banks for which the synthesis filters are time-reversed versions of the analysis filters: $\breve{G}_{j}(z)=G_{j}\left(z^{-1}\right)$ in Fig. 2. As shown in [16], an RFB is equivalent to a uniform $M$-band filter bank, which proves that an RFB can be described by a polyphase matrix $\boldsymbol{\Gamma}(z)$. Moreover, if the RFB is orthonormal, this matrix is paraunitary, i.e., $\boldsymbol{\Gamma}(z) \boldsymbol{\Gamma}\left(z^{-1}\right)^{T}=\mathbf{I}[6]$.

After some algebra, it can be shown that the analysis/synthesis equations corresponding to an orthonormal two-band RFB (notations of Fig. 3) can be written into three sets

$$
\begin{aligned}
& \frac{1}{p q} \sum_{k=0}^{p-1} G\left(\zeta_{q}^{s} \zeta_{p}^{-k} z^{-1}\right) G\left(\zeta_{p}^{k} z\right)=\delta_{s} \\
& \text { for } s=0 \ldots q-1 \\
& \frac{1}{p(p-q)} \sum_{k=0}^{p-1} H\left(\zeta_{p-q}^{s} \zeta_{p}^{-k} z^{-1}\right) H\left(\zeta_{p}^{k} z\right)=\delta_{s} \\
& \text { for } s=0 \ldots p-q-1 \\
& \sum_{k=0}^{p-1} H\left(\zeta_{p-q}^{s} \zeta_{p}^{k} z^{-q}\right) G\left(\zeta_{p}^{k} z^{p-q}\right)=0 \\
& \text { for } s=0 \ldots p-q-1 .
\end{aligned}
$$

Note that if $G(z)$ satisfies (1)-(3), so does $G\left(z^{-1}\right)$. This explains why, in a design problem where the attenuation - which depends only on the modulus of the frequency response of the filter-is minimized, the optimum is usually not unique.

Actually, we shall consider only the first set (1) of equations, which is related to the orthonormality of the "father" limit functions [17]. Once $G$ is designed, it is always possible to recover $H$ (see Section III-C); this filter is even unique when $p-q=1$. Moreover, once $G(z)$ is designed, the attenuation level of the $H$ branch is frozen. This ensues from the power complementary equation

$$
\begin{aligned}
\forall \nu \in \mathbb{R}, \quad \frac{1}{p q} & \sum_{l=0}^{q-1}\left|G\left(e^{2 i \pi \frac{\nu+l}{q}}\right)\right|^{2} \\
& +\frac{1}{p(p-q)} \sum_{l=0}^{p-q-1}\left|H\left(e^{2 i \pi \frac{\nu+l}{p-q}}\right)\right|^{2}=1
\end{aligned}
$$

which is a consequence of orthonormality. For example, let us analyze a sinusoid $e^{2 i \pi k f}$ by the filter bank. Denoting the lowpass and highpass outputs by $x_{n}$ and $y_{n}$, respectively, we have

$$
\begin{aligned}
\sum_{k=0}^{q-1}\left|x_{n+k}\right|^{2} & =\frac{1}{q} \sum_{l=0}^{q-1}\left|G\left(e^{2 i \pi \frac{f+l}{q}}\right)\right|^{2} \\
\sum_{k=0}^{p-q-1}\left|y_{n+k}\right|^{2} & =\frac{1}{p-q} \sum_{l=0}^{p-q-1}\left|H\left(e^{2 i \pi \frac{f+l}{p-q}}\right)\right|^{2}
\end{aligned}
$$

where $\sum_{k=0}^{q-1}\left|x_{n+k}\right|^{2}+\sum_{k=0}^{p-q-1}\left|y_{n+k}\right|^{2}=p$. Now, if the lowpass branch is selective, which happens when $G(z)$ minimizes the attenuation (6) defined in Section III, then $G(1) \approx \sqrt{p q}$. Thus, $\sum_{k=0}^{q-1}\left|x_{n+k}\right|^{2} \approx p$ when $f \in\left[-\frac{q}{2 p}, \frac{q}{2 p}\right]$ (passband of $G\left(e^{2 i \pi \nu / q}\right)$ ). Using the power complementary relation, this implies that the lowpass energy that goes through the highpass branch is small. The additional degree of freedom provided by the (possibly nonunique) choice of $H$ is thus neutral as to the selectivity of the $H$ branch.

In contrast with the dyadic case, the orthonormality condition consists of several (actually q) equations; see (1). This explains why the tricks used in the dyadic case to solve various problems (Chebyshev attenuation minimization [4], maximum number of regularity factors [12]) do not hold in the rational case. However, regarding regularity, it can easily be proved that if $G(z)$ is divisible by $N$ factors $\frac{z^{-p}-1}{z^{-1}-1}$, then $\left(\frac{z^{-q}-1}{z^{-1}-1}\right)^{N}$ divides $G(z)$ as well; this constraint is obviously always satisfied in the integer case.

\section{Design AlgORITHM}

We consider the following problem: Find the most selective filter $G(z)$ satisfying the orthonormality equations (1) and, possibly, additional linear constraints (e.g., regularity). The quality of the selectivity will be quantified by a measure of the difference between the moduli of $G(z)$ and of an ideal filter $F(z)$ on the unit circle. The normalized frequency response of $F(z)$ is such that $\left|F\left(e^{2 i \pi \nu}\right)\right|=\sqrt{p q}$ if $\nu \in\left[-\frac{1}{2 p}, \frac{1}{2 p}\right]$, and 0 elsewhere; thus, if $G(z)=F(z)$, the whole rational branch discards the frequencies above $\frac{q}{2 p}$. However, other filters could do the job: They would not necessarily be lowpass since, in the upsampled domain, the lowpass energy of the input signal lies not only around frequency 0 but also near $1 / q, 2 / q \ldots$ We choose, for the sake of simplicity, for $F(z)$ to be a template for $G(z)$. Yet, we surmise that it is necessary for $G(z)$ to be lowpass in order for the iterations to be stable.

The first $(s=0)$ orthonormality relation (1) yields the power complementary equation

$$
\sum_{k=0}^{p-1}\left|G\left(e^{2 i \pi(\nu+k) / p}\right)\right|^{2}=p q .
$$

This shows that the values of $\left|G\left(e^{2 i \pi \nu}\right)\right|^{2}$ in the attenuation band $\left[\frac{1}{2 p}+\epsilon, \frac{1}{2}\right]$ determine uniquely the value of $\left|G\left(e^{2 i \pi \nu}\right)\right|^{2}$ in the passband $\left[0, \frac{1}{2 p}-\epsilon\right]$ for some positive number $\epsilon$. Thus, it is sufficient to minimize $G(z)$ on its attenuation band in order to minimize the difference between the moduli of $G(z)$ 
and $F(z)$ over the whole spectrum. Of course, such a property would not hold in the biorthonormal case. We choose the $\mathbf{L}^{2}$ norm to quantify the attenuation

$$
a_{\nu_{0}}(G)=\int_{\nu_{0}}^{1 / 2}\left|G\left(e^{2 i \pi \nu}\right)\right|^{2} d \nu
$$

for $\nu_{0}=\frac{1}{2 p}+\epsilon>\frac{1}{2 p}$ because of its many appealing mathematical properties.

As usual in filter design issues, a tradeoff has to be found between the attenuation level and the width $2\left(\nu_{0}-\frac{1}{2 p}\right)$ of the transition band.

\section{A. Description}

Our design algorithm is based on three key ideas.

- The attenuation to minimize is quadratic in $G$, and, provided that the constraints are linear, this amounts to solving a linear system of equations.

- The orthonormality constraints (1) are quadratic in $G$ but are linear in $G$ if we consider biorthonormality constraints instead.

- The orthonormality constraints (1) are degenerated and contain approximately twice as many equations as needed, due to the symmetry $z \rightarrow z^{-1}$

These observations suggest a recursive procedure to compute a sequence $\left\{G_{l}(z)\right\}_{l \in \mathbb{N}}$ of filters such that $G_{\infty}(z)$ is the designed filter: $G_{l-1}$ being given, minimize $a_{\nu_{0}}(G)$ for $G$ under the linear constraints

$$
\begin{aligned}
& \frac{1}{p q} \sum_{k=0}^{p-1} G_{l-1}\left(\zeta_{q}^{s} \zeta_{p}^{k} z^{-1}\right) G\left(\zeta_{p}^{k} z\right) \\
& \quad=\delta_{s}+\text { strictly positive powers of } z
\end{aligned}
$$

for $s=0 \ldots q-1$, i.e., roughly half the biorthonormality constraints; this provides $G=G_{\text {min }}$. Then, we let

$$
G_{l}(z)=\frac{G_{l-1}+G_{\min }}{2}
$$

which prevents convergence toward a nonorthonormal solution. At each iteration step $l$, a reconstruction error is computed, and the iterations stop when this error is small enough. We now come to the details of the implementation.

\section{B. Implementation}

1) Quadratic Functional: First, we identify $G(z)$ with the column vector $G$ of its coefficients $\left[g_{0}, g_{1}, \ldots g_{N}\right]^{t}$, where $N=\operatorname{deg}(G(z))$. Thus, (6) takes the form

$$
a_{\nu_{0}}(G)=G^{t} \mathbf{A}_{0} G
$$

where the symmetric positive definite Toeplitz matrix $\mathbf{A}_{0}$ is defined by $\left[\mathbf{A}_{0}\right]_{i, j}=-\frac{\sin 2 \pi(i-j) \nu_{0}}{2 \pi(i-j)}$ if $0 \leq i \neq j \leq N$ and $\left[\mathbf{A}_{0}\right]_{i, i}=1 / 2-\nu_{0}$ for $i=0 \ldots N$.
2) Constraints: Second, we rewrite half of the reconstruction equations.

For practical reasons of accuracy [because (1) contains complex irrational numbers], we use an equivalent polyphase formulation of (1)

$$
\begin{aligned}
\sum_{k} g_{-n_{0}^{\prime} p}^{q}[k-n p] g_{-n_{0} p}^{q}[k]=\delta_{n_{0}^{\prime}-n_{0}} \delta_{n} \\
\left\{\begin{array}{l}
\forall n \in \mathbb{Z} \\
\forall n_{0}, n_{0}^{\prime}=0 \ldots q-1 .
\end{array}\right.
\end{aligned}
$$

The degeneracy resulting from the symmetry $\left(n_{0}, n_{0}^{\prime}, n\right) \rightarrow$ $\left(n_{0}^{\prime}, n_{0},-n\right)(10)$ is removed by considering the subset $\mathbb{K}$ of index values defined by

$$
\begin{aligned}
\mathbb{K}=\left\{\left(n_{0}, n_{0}^{\prime}, n\right) \in[0 \ldots q-1]^{2} \times \mathbb{N}\right. \text { s.t. } \\
\left.\qquad \begin{array}{l}
\text { if } n=0 \text { then } 0 \leq n_{0} \leq n_{0}^{\prime} \leq q-1 \\
\text { if } n \geq 1 \text { then } n_{0}, n_{0}^{\prime}=0 \ldots q-1
\end{array}\right\} .
\end{aligned}
$$

The working idea of our algorithm is thus to enforce the following linear constraints on $G(z)$.

$$
\begin{aligned}
& \sum_{k} \gamma_{-n_{0}^{\prime} p}^{q}[k-n p] g_{-n_{0} p}^{q}[k]=\delta_{n_{0}^{\prime}-n_{0}} \delta_{n} \\
& \qquad \text { for }\left(n_{0}, n_{0}^{\prime}, n\right) \in \mathbb{K} .
\end{aligned}
$$

Here, $\gamma[n]$ is substituted for $g_{l-1}[n]$ ( $l=$ iteration step) to avoid too many indices.

This set of equations is rewritten in vector notation as

$$
\mathbf{S} G=C
$$

where we have denoted by $C$ a constant column vector and by $\mathbf{S}$ the rectangular constraint matrix. The coefficients of this matrix are those of $G_{l-1}$, and actually, $\mathbf{S}$ is linear with respect to $G_{l-1}$.

The number $N_{\mathrm{S}}$ of rows of $\mathbf{S}$ is directly computed in the software by simply counting the number of constraints. It can, in fact, be expressed exactly in function of $N, p$, and $q$ but yields such an intricate form that it would be pointless to write it down here.

Other linear (with respect to $G$ ) conditions can be added, such as the regularity constraints. For instance, $K$ regularity factors imply that $\left(\frac{z^{p}-1}{z-1} \frac{z^{q}-1}{z-1}\right)^{K}$ divides $G(z)$, which is equivalent to

$$
\left.\begin{array}{rl}
\sum_{n}(k+n p)^{r} g_{k}^{p}[n] & =\sum_{n}(n p)^{r} g_{0}^{p}[n] \quad \forall 1 \leq k \leq p-1 \\
\sum_{n}(k+n q)^{r} g_{k}^{q}[n] & =\sum_{n}(n q)^{r} g_{0}^{q}[n] \quad \forall 1 \leq k \leq q-1 \\
\text { for } r=0 \ldots K-1
\end{array}\right\}
$$

or under vector form

$$
\mathbf{T} G=0 .
$$

In this equation, $\mathbf{T}$ is an $N_{\mathbf{T}} \times(N+1)$ rectangular matrix, where $N_{\mathbf{T}}=K(p+q-2)$. 
3) The Minimization: Using Lagrange's method, our constrained minimization problem can be rewritten into an unconstrained form. Introducing the vector multiplier $\lambda$ of size $\left(N_{\mathbf{S}}+N_{\mathbf{T}}\right) \times 1$, we have to minimize the function $J_{0}(G)=$ $G^{t} \mathbf{A}_{0} G-2 \lambda^{t} \mathbf{\Sigma} G$, where $\boldsymbol{\Sigma}$ is the global constraint matrix $\boldsymbol{\Sigma}=\left[\begin{array}{ll}\mathbf{S}^{t} & \mathbf{T}^{t}\end{array}\right]^{t} \cdot \lambda$ is then determined by enforcing (13) and (15).

The minimization of $J_{0}(G)$ amounts to solving, for $X$, an equation of the form $\mathbf{A}_{0} X=Y$. However, $\mathbf{A}_{0}$ is ill conditioned when $N$ is large enough so that a "stabilization" trick has to be devised. In this purpose, we add another quadratic term to $J_{0}(G)$, namely, $\|\mathbf{S} G-C\|^{2}$, which obviously does not change the final result $G_{\min }$. For conditioning reasons, we are thus led to minimize the function of $G$

$$
J(G)=G^{t} \mathbf{A}_{0} G+\left(G^{t} \mathbf{S}^{t}-C^{t}\right)(\mathbf{S} G-C)-2 \lambda^{t} \mathbf{\Sigma} G .
$$

The result is

$$
\begin{aligned}
\lambda & =\left(\mathbf{\Sigma M}^{-1} \boldsymbol{\Sigma}^{t}\right)^{-1}\left[\begin{array}{c}
C \\
0_{N_{\mathrm{T}}}
\end{array}\right]-\left[\begin{array}{c}
C \\
0_{N_{\mathbf{T}}}
\end{array}\right] \\
G_{\min } & =\mathbf{M}^{-1}\left(\boldsymbol{\Sigma}^{t} \lambda+\mathbf{S}^{t} C\right)
\end{aligned}
$$

where $\mathbf{M}=\mathbf{A}_{0}+\mathbf{S}^{t} \mathbf{S}$ is a much better conditioned matrix than $\mathbf{A}_{0}$.

4) To Summarize: The algorithm as it is currently implemented (MATLAB, MacIntosh Quadra 840) runs through the following steps.

1) Choose an initial filter $G_{0}$ of degree $N$. It turns out that since the algorithm is completely insensitive to the choice of the first filter, the initialization method does not really matter.

2) Compute the constant parameters of the algorithm such as $\mathbf{T}, \mathbf{A}_{0}$, and $C$.

3) At iteration step $l$, we assume that $G_{l-1}$ is known. Compute $\mathbf{S}$ with (13), and obtain $G_{\min }$ according to (17).

4) Update $G_{l}$ according to (8), and compute the reconstruction error $\varepsilon(\cdot)$ defined by

$$
\varepsilon(G)=\sup _{n, n^{\prime} \in \mathbb{Z}}\left|\sum_{k} g_{n^{\prime} p}^{q}[k] g_{n p}^{q}[k]-\delta_{n^{\prime}-n}\right| .
$$

If $\varepsilon(G)$ is greater than or equal to some small predefined value (e.g., $10^{-11}$ ), go to Step 3 ; else exit with $G_{\text {designed }} \approx G_{l}$.

5) Convergence: The mathematical convergence of this algorithm is still an open problem, and we are not going to answer it here. However, the overwhelming agreement between our observations (setting a $10^{-11}$ threshold for the perfect reconstruction error) leads us to claim the following.

- If $q=1$, convergence is unconditional; but increased regularity order (above 5 for small filters and higher orders for larger ones) requires increased computing accuracy.

- if $q>1$, convergence is unconditional if no more than one regularity order is required.

- Convergence (i.e., rate and limit) is independent of the initial filter $G_{0}$, although an adequate choice may reduce the number of iterations.
- The convergence rate is exponential: $\varepsilon \approx 0.7^{l}$. This is not a particularly surprising result since the algorithm is a kind of fixed-point algorithm, which usually shows this behavior.

- Although $N$ is arbitrary above $N_{\mathbf{T}}+N_{\mathbf{S}}-1$ (this ensures that there are at least as many unknowns as constraints), the resulting filter always ends up with degree $N_{0}$, where $N-p<N_{0} \leq N$, and $N_{0} \equiv-1 \bmod p$. Actually, the coefficients between $N_{0}+1$ and $N$ are below computing accuracy, and choosing $N$ or $N_{0}$ does not change the result. The degree of $G(z)$ can thus be restricted to be of the form $k \times p-1$.

\section{Highpass Filter}

The highpass filter will be deduced from the lowpass. In the dyadic case, for example, we have the formula $H(z)=$ $\pm z^{-2 n-1} G\left(-z^{-1}\right)$, which can be extended to the rational case, provided $p-q=1$; this is shown in the following subsection.

1) Direct Method: Assume $p-q=1$, and consider (1)-(3). We can rewrite them into matrix form $\boldsymbol{\Gamma}(z) \overline{\boldsymbol{\Gamma}}\left(z^{-1}\right)^{t}=\mathbf{I}$, where

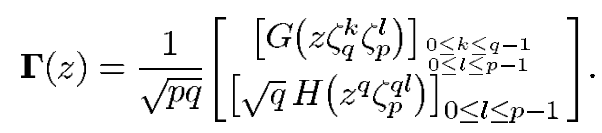

The perfect reconstruction hypothesis implies that $\operatorname{det}(\boldsymbol{\Gamma})$ is a pure delay. Specifically, this delay is of the form $z^{-\left(n+\frac{1}{2}\right) p q}$ up to the multiplication by a constant of modulus 1 for some integer $n$; this is because the change $z \rightarrow z e^{\frac{2 i \pi}{p q}}$ rotates the $p$ columns and the first $q$ rows of $\boldsymbol{\Gamma}(z)$ by 1 , and this actually results in the multiplication of $\operatorname{det}(\boldsymbol{\Gamma}(z))$ by -1 .

Denote $|\boldsymbol{\Gamma}|_{k, l}$ as the minor of order $k, l$ of $\boldsymbol{\Gamma}$, i.e., the determinant of the $p-1 \times p-1$ matrix built from $\boldsymbol{\Gamma}$ without line $k$ and column $l$. The classical matrix inversion theorem states

$$
\boldsymbol{\Gamma}^{-1}=\frac{1}{\operatorname{det} \boldsymbol{\Gamma}}\left[(-1)^{k+l}|\boldsymbol{\Gamma}|_{l, k}\right]_{0 \leq k, l \leq p-1} .
$$

Applied to our case, this provides

$$
H\left(z^{q}\right)=z^{-\left(n+\frac{1}{2}\right) p q}\left|\overline{\boldsymbol{\Gamma}}\left(z^{-1}\right)\right|_{p-1,0}
$$

up to the multiplication by a constant of modulus 1 . Once again, it is easy to verify directly that the right-hand side is truly polynomial in $z^{q}$ by making the change $z \rightarrow z e^{2 i \pi / q}$. The $|\boldsymbol{\Gamma}|$ term is multiplied by $(-1)^{p}$ (rotation of column vectors in $\left.|\boldsymbol{\Gamma}|_{p-1,0}\right)$, which is cancelled by the change of the delay term. As in the dyadic case, the right-hand side depends only on $G(z)$, which shows that $G$ determines $H$ uniquely (up to a delay).

This exact result is, however, not very practical because of the following problem: If $G$ is of degree $N$, (20) implies that $H$ is of degree $N$ as well, whereas by other means, we know that the true degree of $H$ is (approximately) $\frac{N}{q}$. An alternative method, based on the factorization of paraunitary matrices [6], is more reliable once the reconstruction error is kept small enough. 
2) Via the Lossless Cascade: We do not assume $p-q=1$ anymore. The analysis/synthesis equations from (10) can be put into matrix form [17], resulting in the polyphase matrix $\boldsymbol{\Gamma}_{P}$ of the filter bank being paraunitary. Specifically, we have [6] $\boldsymbol{\Gamma}_{P}(z) \boldsymbol{\Gamma}_{P}\left(z^{-1}\right)^{t}=\mathbf{I}$, where $\boldsymbol{\Gamma}_{P}$ can be decomposed into two rectangular submatrices

$$
\boldsymbol{\Gamma}_{P}=\left[\begin{array}{l}
\mathbf{G} \\
\mathbf{H}
\end{array}\right]
$$

Here, $\mathbf{G}=\left[\mathbf{G}_{k, l}\right]$ is of size $q \times p$ and depends only on $G(z)$, whereas $\mathbf{H}=\left[\mathbf{H}_{k, l}\right]$ is of size $p-q \times p$ and depends only on $H(z)$. Their respective expressions are

$$
\begin{aligned}
& \mathbf{G}_{k, l}(z)=G_{k p-l q}^{p q}(z) \quad \text { for }\left\{\begin{array}{l}
0 \leq k \leq q-1 \\
0 \leq l \leq p-1
\end{array}\right. \\
& \mathbf{H}_{k, l}(z)=H_{k p-l(p-q)}^{p(p-q)}(z) \quad \text { for }\left\{\begin{array}{l}
0 \leq k \leq p-q-1 \\
0 \leq l \leq p-1 .
\end{array}\right.
\end{aligned}
$$

Note that $G(z)$ and $H(z)$ can be retrieved from $\mathbf{G}$ and $\mathbf{H}$ through

$$
\begin{aligned}
& G(z)=U_{q}\left(z^{p}\right)^{t} \mathbf{G}\left(z^{p q}\right) U_{p}\left(z^{-q}\right) \\
& H(z)=U_{p-q}\left(z^{p}\right)^{t} \mathbf{H}\left(z^{p(p-q)}\right) U_{p}\left(z^{-p+q}\right)
\end{aligned}
$$

where $U_{N}(z)=\left[1, z^{-1}, z^{-2}, \ldots z^{-N+1}\right]^{t}$.

Since $\boldsymbol{\Gamma}_{P}$ is a square paraunitary matrix, it can be (nonuniquely) factorized [6] into the form

$$
\boldsymbol{\Gamma}_{P}(z)=z^{-s} \mathbf{P}_{0} \prod_{k=1}^{M}\left(\mathbf{I}+\left(z^{-1}-1\right) u_{k} u_{k}^{t}\right) .
$$

The parameters of this factorization are chosen as $s p+M=$ $\operatorname{deg}\left(\operatorname{det} \boldsymbol{\Gamma}_{P}(z)\right), \mathbf{P}_{0}$ is a constant orthogonal matrix, and $\left\{u_{k}\right\}_{k=1 \ldots M}$ are unitary $p \times 1$ vectors.

Equation (23) still holds for rectangular paraunitary matrices [18], [29] (for which $\boldsymbol{\Gamma}_{P}(z) \boldsymbol{\Gamma}_{P}\left(z^{-1}\right)^{t}=\mathbf{I}$, where $\boldsymbol{\Gamma}_{P}(z)$ has more columns than rows). The only change is that the constant orthogonal matrix $\mathbf{P}_{0}$ is replaced by a rectangular matrix that satisfies $\mathbf{P}_{0} \mathbf{P}_{0}^{t}=\mathbf{I}$.

Although the product is still finite, $M$ is no longer a constant and depends on the other parameters of the factorization. The vectors $u_{n}$ are computed by induction, using a technique described in [30] (for square matrices) and are also sketched in [18, pp. 67-68].

Thus, computing $H(z)$ amounts to factorizing the rectangular matrix $\mathbf{G}(z)$ into paraunitary components and then adding rows to the rectangular matrix $\mathbf{P}_{0}$ so that it becomes a square orthogonal matrix. In turn, the product (23) will provide the global polyphase matrix of the system $\boldsymbol{\Gamma}_{P}(z)$ from which $G(z)$ and $H(z)$ can be extracted.

Here, we see some advantages of this method.

- The filter bank has exact perfect reconstruction, whereas, out of the design algorithm, $G(z)$ is only almost orthonormal $\left(\varepsilon \approx 10^{-11}\right)$.

- The degree of $H(z)$ is now (approximately) $\frac{N}{q}$.

- The factorization provides an economic way to describe the two-band system. Only $p^{2}+\frac{N}{q}$ real numbers are required for the whole system to be compared with the $\left(1+\frac{1}{q}\right) N+2$ numbers by the direct method. This computation comes from the (experimental) observation that exactly $\left\lceil\frac{N}{p q}\right\rceil$ paraunitary sections are sufficient to decompose $\boldsymbol{\Gamma}_{P}$ when $G(z)$ is obtained from our algorithm.

- The factorization (23) is nicely adapted to the implementation of filter banks in fixed precision [6] while retaining the perfect reconstruction feature; the trick amounts to using non-normalized versions, with integer coefficients, of the unitary vectors $u_{k}$ in (23). As indicated in [6], the orthogonal matrix $\mathbf{P}_{0}$ can itself be put into the similar form

$$
\mathbf{P}_{0}=\prod_{k=1}^{p}\left(\mathbf{I}-2 v_{k} v_{k}^{t}\right)
$$

where the vectors $v_{k}$ are unitary.

We shall thus use this economic description in Section IV for the presentation of our design results. Note that our method requires the lowpass filter to show a very low reconstruction error. This is always the case for our algorithm when we set the maximum reconstruction error to be less than $10^{-11}$, although, for very large filters, a greater accuracy may be needed.

When $p-q \geq 2$, the matrix $\mathbf{P}_{0}$ has to be filled by more than one row. Thus, $H(z)$ is no longer unique, but as shown in Section III-B, this degree of freedom does not change the selectivity of the highpass branch, which only depends on the lowpass. This means that any filter $H(z)$ obtained will meet our requirements.

\section{DESIGN RESUlts AND COMPARISONS}

This section provides some design examples. We compare our filters with those obtained by other existing methods. Quite a few design algorithms are now classical in the dyadic orthonormal case [3], [4], [13], [14], [31] as well as in the dyadic biorthonormal case [9], [32]. In contrast, much less [16], [22, orthonormal], [32, biorthonormal] has been done in the true rational case.

We shall first compare our new algorithm with others in the dyadic case. Then, for $p / q=3 / 2$, we shall see that our designed filter shows better characteristics than Kovačević's [16], [22], whose method is based on a direct minimization for the parameters of the cascade decomposition [6], [30] of the filter bank.

Finally, we shall exhibit a set of filters for $p / q=6 / 5$. This factor is especially adapted to audio processing because it is very close to the Bark scale factor, which is a psychophysical frequency unit [33]. Actually (psychoacoustic), coding attempts [24] using these filters proved quite promising.

\section{A. Comparison with the Dyadic Case}

Our algorithm is able to provide filters for any value of $p$ and $q(p>q)$, and, in particular, for $p / q=2 / 1$. Depending on the attenuation measure, various orthonormal filter design algorithms exist. For the $\mathbf{L}^{\infty}$ norm (a Chebyshev approximation problem), solutions are worked out in [3], [4, no regularity], and [14, with regularity]; for the $\mathbf{L}^{2}$ norm, [31] provides a method that uses linear programming.

The orthonormality equations and the attenuation measure involve only the filter $R(z)=G(z) G\left(z^{-1}\right)$, and thus, the 

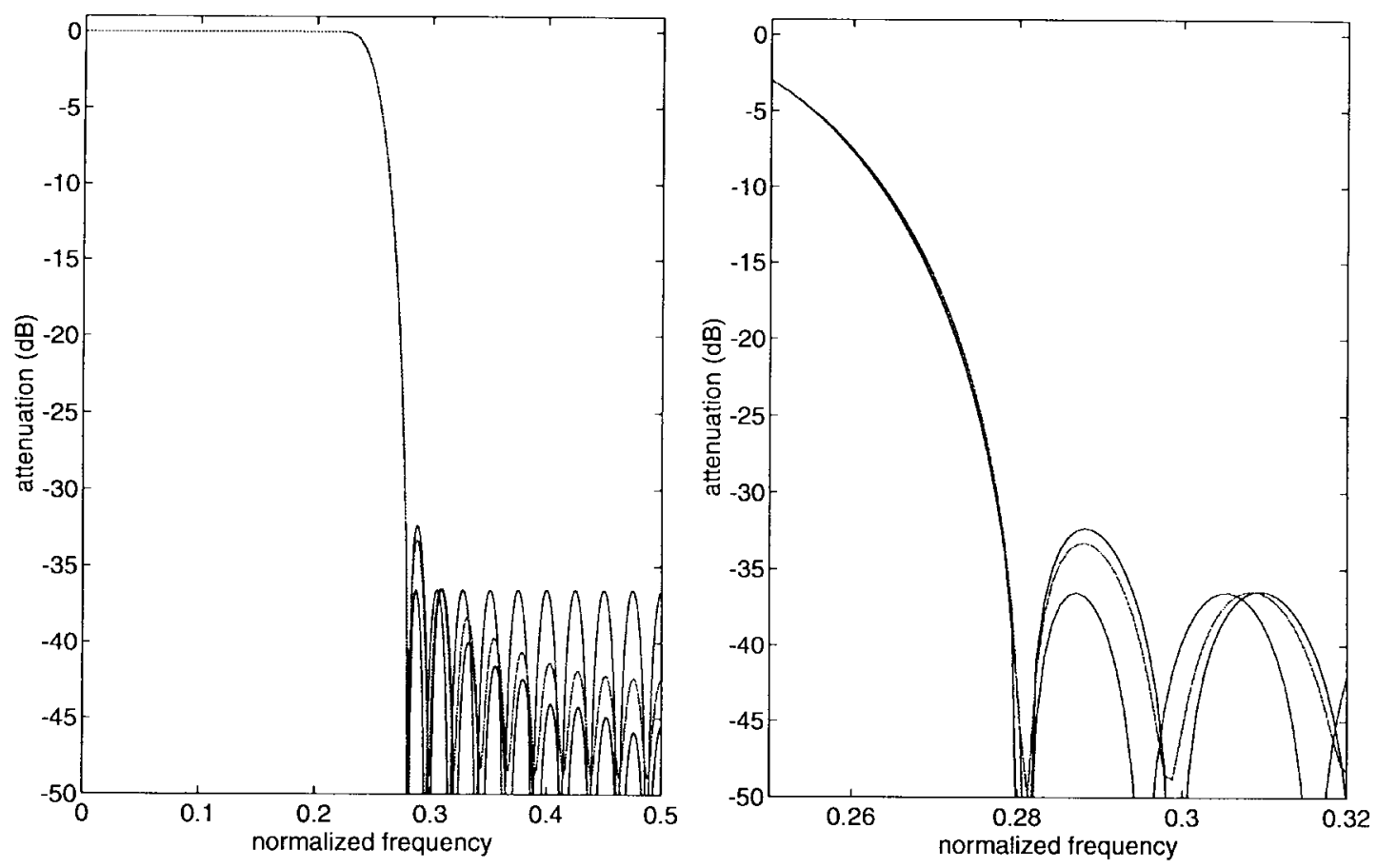

Fig. 4. Frequency responses of the three filters described in Section IV-A. Full scale (left) and closeup around the transition frequency (right).

minimization process provides only $R(z)=R_{\min }(z)$. A consequence is that any filter $G(z)$ satisfying $G(z) G\left(z^{-1}\right)=$ $R_{\min }(z)$ is a solution of the minimization problem, implying that several filters are minimal. In contrast, our algorithm provides only one filter. Experiments show that it is always the one that has all its roots inside the unit circle.

We have thus computed filters of degree 39 for a (normalized) $\mathbf{L}^{\infty}$ transition frequency of 0.279 . This compares with a transition frequency of 0.275 for the $\mathbf{L}^{2}$ norm and $\nu_{0}=0.2754$ for our algorithm. Fig. 4 shows that the first ripple is highest for the $\mathbf{L}^{2}$ solution, followed by our algorithm, and then by the Mintzer/Smith-Barnwell (MSB) solution. There is a difference of approximately $3 \mathrm{~dB}$ between our solution and MSB's and $1 \mathrm{~dB}$ with the quadratic solution. These differences are obviously very weak. Actually, we have always observed that the solution of our algorithm performs slightly better than the $\mathbf{L}^{2}$ solution and slightly worse than the $\mathbf{L}^{\infty}$ solution.

The convergence has been achieved within 78 iterations, which take approximately $37 \mathrm{~s}$ on a Macintosh Quadra 840 under MATLAB. The convergence of the reconstruction error plotted in Fig. 5 is almost exactly exponential after 30 iterations. A very accurate computation of the convergence rate yields a value between 0.7366 and 0.7367 .

\section{B. Comparison in the Case $p / q=3 / 2$}

Rational design examples are rare in the literature. Kovačević and Vetterli [16], [22] described a method (orthonormal case) that is essentially based on the lossless decomposition of the polyphase matrix [6]. They designed a lowpass filter of length 32 and of transition frequency approximately 0.21 . Actually, their algorithm was not fully optimized since they essentially wanted to show the advantage of direct instead of indirect design. In order to compare our algorithm with theirs, we set the length of our filter to 30 and $\nu_{0}$ to 0.208 , thus ensuring the same "real" transition frequency. Our algorithm converged after 72 iterations in 28 $\mathrm{s}$, the exponential convergence rate lying between 0.6824 and 0.6825 . The lowpass and highpass filters designed by both algorithms are plotted in Fig. 6. It is evident that our algorithm has a much better attenuation (the difference amounts to $12 \mathrm{~dB}$ ).

\section{Example $p / q=6 / 5$ and $N=203$}

The last example is justified by the critical band phenomenon brought to light by psychoacoustic experiments [33]. We showed [24] the advantage of the $6 / 5$ scale factor for large band audio coding.

In order to ensure a good frequency selectivity up to the 19th iteration (19 iterations span five octaves, i.e., the frequency interval over which the Bark scale is almost logarithmic), a rather long lowpass filter is required. Here, the behavior of the iterations is not negligible, as far as shift error (closely connected to the selectivity of the iterated filter bank [18]) and regularity [28] are concerned.

Since our algorithm is fast and unsupervised, we took the opportunity to try various transition frequencies and filter lengths. It appeared that the choice $\nu_{0}=0.093$ (for the ideal filter $\nu_{0}=\frac{1}{2 p} \approx 0.083$ ), degree $N=204$, and one regularity factor was suitable for our application.

The design algorithm converged after 81 iterations and approximately in $36 \mathrm{~min}$, whereas the reconstruction error decreased exponentially at the rate of $0.736^{\text {iteration }}$. The lowpass and highpass filters are plotted in Fig. 7.

For the reasons discussed in Section III-C2, we use the lossless decomposition to express the filters obtained by our algorithm. They are given in Table I, where the vectors are 


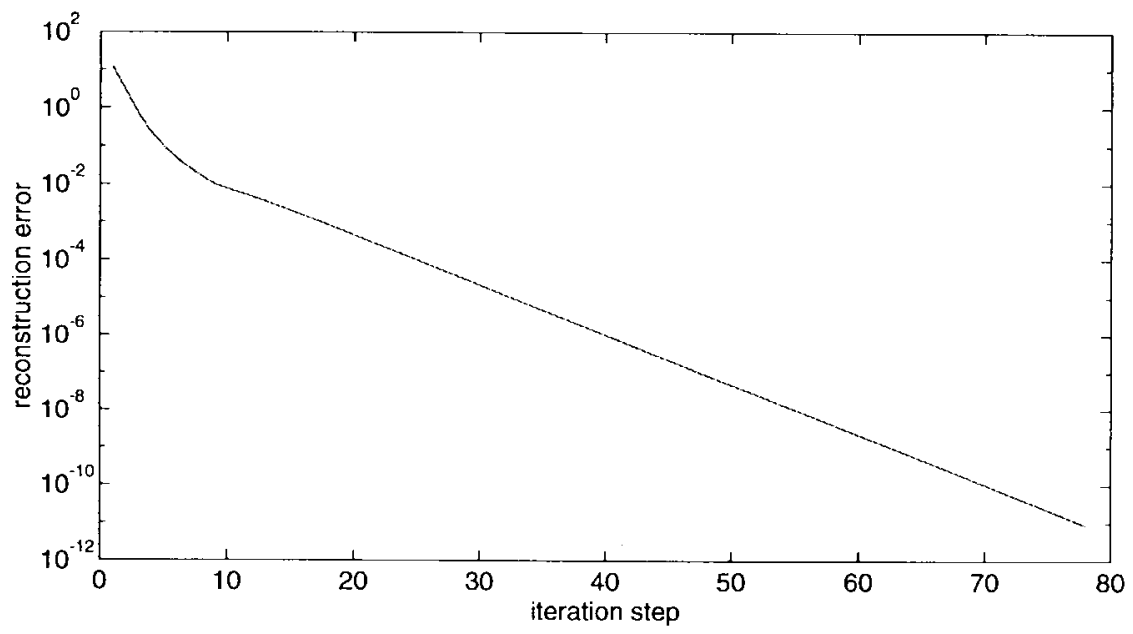

Fig. 5. Convergence of the algorithm for the case IV-A.
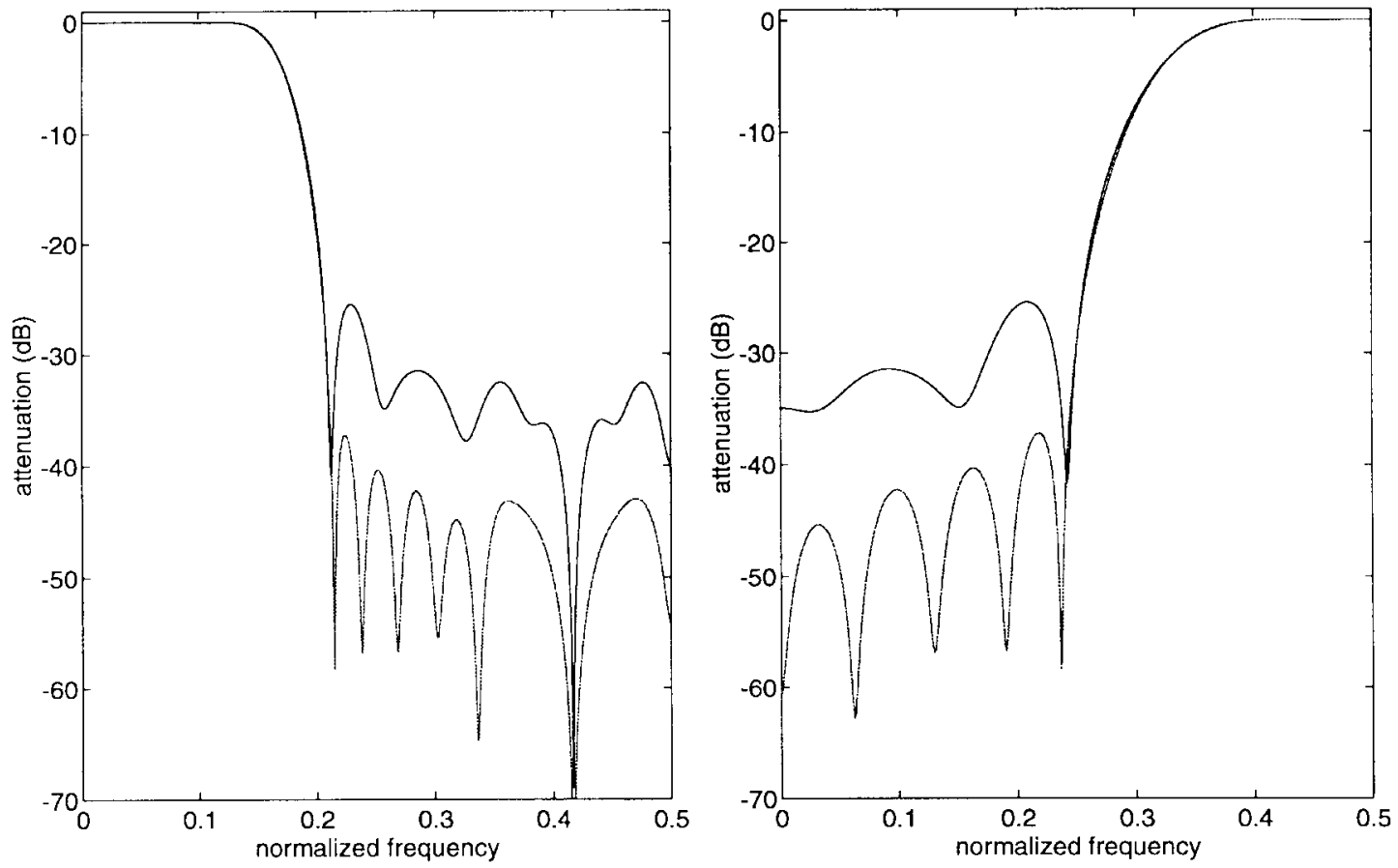

Fig. 6. Frequency responses of the filters obtained by the following algorithm (case IV-B) and by the lossless factorization [16], [22]. Lowpass (left) and highpass (right) filters.

non-normalized versions of the vectors $u_{k}$ (23) and $v_{k}$ (24). Notice that quantizing $\mathbf{P}_{0}$ removes the regularity factor from the lowpass filter; in spite of that, the values of the quantized filter at these frequencies is small enough (approximately $10^{-5}$ ) to prevent any divergence resulting from the lack of regularity, given that we make 19 iterations.

To be complete, we computed the limit functions $\varphi_{n}$ generated by these filters [17]. Here, the functions are almost shift invariant, i.e., $\varphi_{n}(x) \approx \varphi_{0}(x-n)$. The shift error is approximately 0.004 [18], [34]. We have thus plotted only one of them (see Fig. 8) together with the corresponding "mother" wavelet (i.e., highpass). The theoretical support of both functions is approximately the degree of $G$ (see [17]), i.e., 203. In practice, however, these functions almost cancel outside intervals of size 40 and 50 respectively.

\section{Remarks}

The filters designed by the proposed algorithm have excellent attenuation characteristics. This is particularly obvious in the few cases where optimal solutions are available.

As far as iterations are concerned, regularity is a more important matter than for dyadic schemes. Basically, more iterations are required in the rational case. Although the rational schemes may arguably diverge more slowly than the dyadic when $G(z)$ is not regular (because $p / q$ is closer to 1 ), we advise at least one regularity factor to ensure the stability of the frequency response of the iterated lowpass filter.

Unfortunately, except in the integer $q=1$ case, our algorithm diverges almost surely when more than one regularity factor is required, and because of this divergence, we are also 


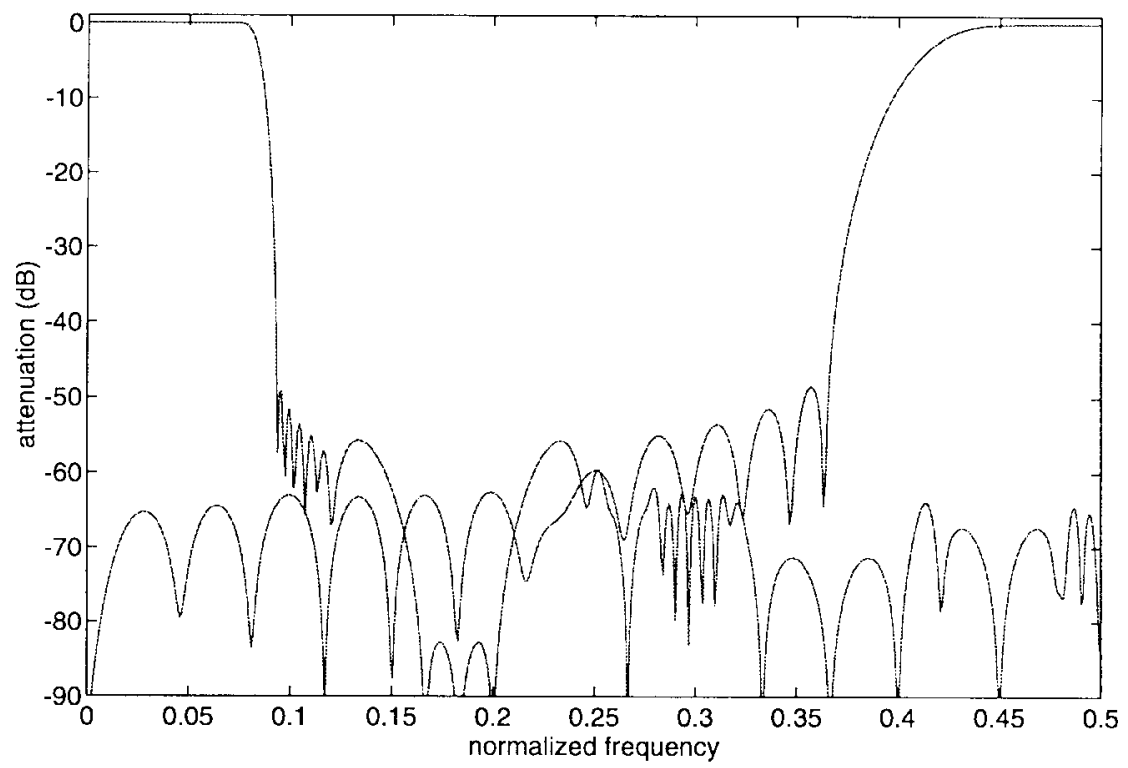

Fig. 7. Frequency responses of the filters obtained in case IV-C. Lowpass (length $=204$ ) and highpass $($ length $=41)$ filters.
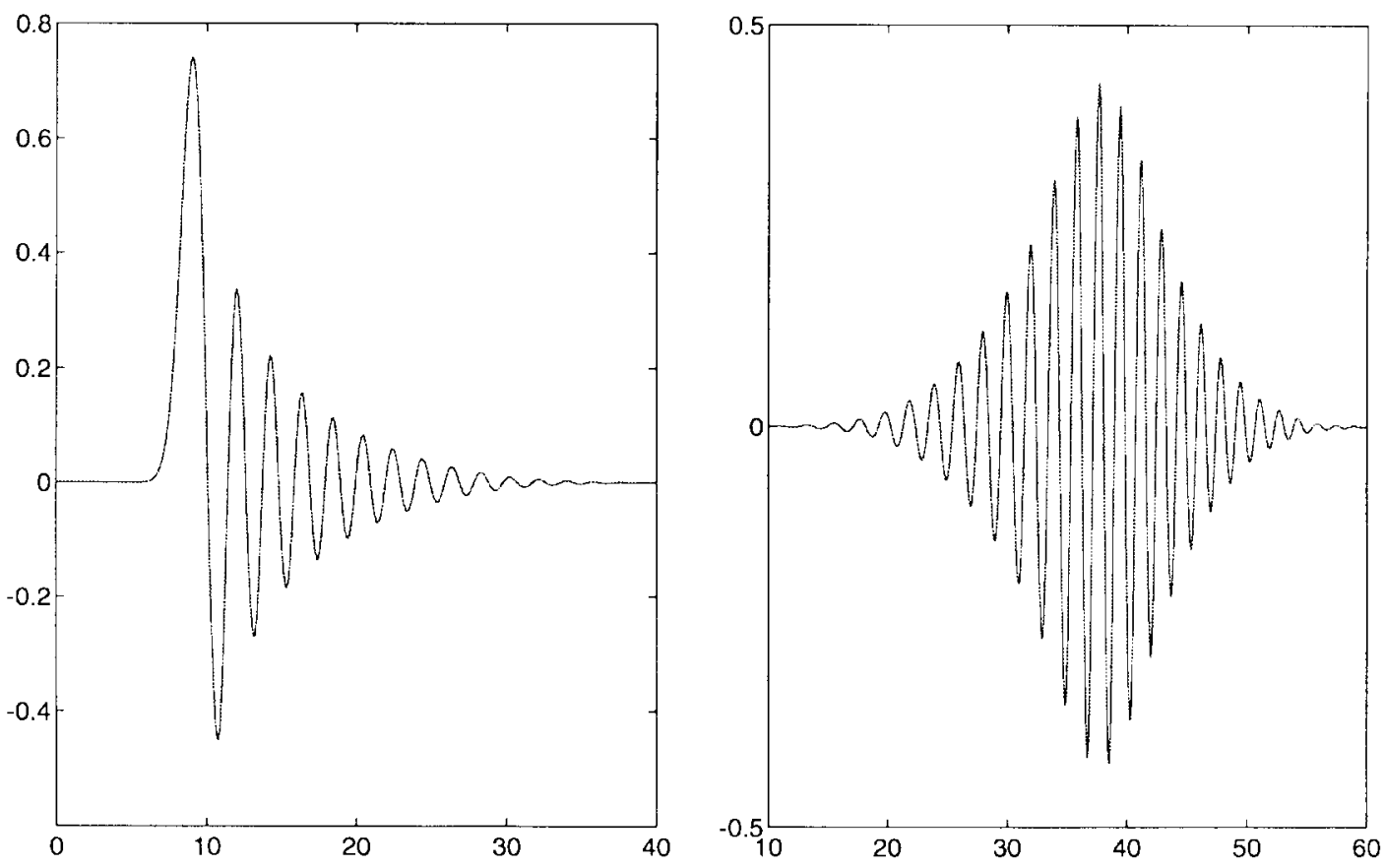

Fig. 8. Father (left) and mother (right) wavelets generated by the filters of case IV-C.

unable to compute the rational equivalents of Daubechies' filters [12]. A full account of the advantages of regularity is thus left unanswered at that point. In the dyadic case, a study [35] highlighted its potential for image coding.

Finally, as noticed in case IV-C, the effective support of the limit wavelets is much smaller than their theoretical support. This observation has long been known for dyadic wavelets [12]. Let us explain and quantify this characteristic. The attenuation level of a filter $G(z)$ being given, it is experimentally observed [36] that its transition bandwidth $\delta \nu$ is (roughly) inversely proportional to its degree: $N \delta \nu \approx C$ if we let $N=\operatorname{deg}(G(z))$. On the other hand, iterating $j$ times yields [17] $G_{j}(z)=G\left(z^{p^{j-1}}\right) G\left(z^{q p^{j-2}}\right) \ldots G\left(z^{q^{j-1}}\right)$. Under a regularity hypothesis, this iterated filter is lowpass, and its frequency support is close to the ideal $\left[-\frac{1}{2 p^{j}}, \frac{1}{2 p^{j}}\right]$. Besides, the transition bandwidth of $G_{j}(z)$ is $\delta \nu_{j}=\frac{\delta \nu}{p^{j-1}}$, and its degree is $N_{j}=N \frac{p^{j}-q^{j}}{p-q}$, which implies that $\frac{p-q}{p} N_{j} \delta \nu_{j} \rightarrow C$ as $j$ tends to infinity. Thus, $G_{j}$ has the same design characteristics as an optimal filter of degree $\frac{p}{p-q}$ times smaller. Since $G_{j}$ is very close to an ideal filter, we conclude that its coefficients almost cancel (out of an interval of length $N_{j} \frac{p-q}{p}$ ), and this explains why the support of the limit lowpass function is approximately included in an interval of size $\frac{N}{p}$.

It is thus because of the iterations and of the selectivity constraint that an iterated filter bank loses a part of 
TABLE I

Results for the CASE IV-C: Description by the (Non-Normalized) Parameters of (23) AND (24)

\begin{tabular}{r|r|r|r|r}
\hline \multicolumn{5}{c}{$\mathbf{P}_{0}$} \\
\hline \multicolumn{1}{c|}{$\mathrm{v}_{1}$} & \multicolumn{1}{|c|}{$\mathrm{v}_{2}$} & \multicolumn{1}{c|}{$\mathrm{v}_{3}$} & \multicolumn{1}{c}{$\mathrm{v}_{4}$} & \multicolumn{1}{c}{$\mathrm{v}_{5}$} \\
\hline-70734 & 0 & 0 & 0 & 0 \\
23869 & -70869 & 0 & 0 & 0 \\
55675 & 46536 & -80228 & 0 & 0 \\
-21414 & 40972 & 53091 & -73221 & 0 \\
6445 & -23857 & 22899 & 66486 & -37641 \\
28760 & -23753 & 14849 & -14774 & 92645 \\
\hline
\end{tabular}

\begin{tabular}{r|r|r|r|r|r|r}
\hline \multicolumn{7}{c}{ Vectors of the lossless factorization } \\
\hline \hline $\mathrm{u}_{1}$ & \multicolumn{1}{c|}{$\mathrm{u}_{2}$} & \multicolumn{1}{c}{$\mathrm{u}_{3}$} & \multicolumn{1}{c|}{$\mathrm{u}_{4}$} & \multicolumn{1}{c|}{$\mathrm{u}_{5}$} & $\mathrm{u}_{6}$ & $\mathrm{u}_{7}$ \\
\hline-40520 & -42634 & -33906 & -48059 & -23538 & -28 & 0 \\
41126 & 40059 & 40627 & 35260 & 42460 & 479 & 0 \\
-41720 & -37055 & -49555 & -18513 & -61601 & -4455 & 0 \\
41657 & 36465 & 52392 & 13719 & 59329 & 25498 & 0 \\
-39817 & -44374 & -31302 & -56285 & 14705 & -96591 & 0 \\
40069 & 43655 & 31929 & 52423 & 10599 & 1 & 1 \\
\hline
\end{tabular}

its computational efficiency, This is the price we pay for a recursive, low-memory storage, flexible (e.g., addition of new branches) implementation of a constant $Q$ analysis. This relative inefficiency may be relieved by the direct design of a close $M$-band nonuniform filter bank [19]. In this case, however, structural perfect reconstruction is most often lost [19], [20], the storage cost for the filters is heavy, and the flexibility is much reduced.

\section{CONCLUSION}

The new design algorithm we have presented has the following properties.

- It is recursive, and each iteration amounts to solving a simple linear system of equations.

- It is fast, due to its exponential convergence.

- It is automatic (independence from the initialization).

- It can be adapted to various needs by adding new linear constraints or by changing (e.g., weighting) the quadratic attenuation measure.

- It provides excellent, nonequiripple, filters, as shown by the comparison with optimal results in the dyadic case.

Furthermore, as suggested by a reviewer, the universality of the main trick used here-i.e., keeping half of the orthonormality equations-makes it potentially useful for design issues other than rational filter banks.

\section{ACKNOWLEDGMENT}

The author would like to thank the anonymous reviewers and Dr. J. Kovačević for their suggestions. Many thanks are also due to Dr. P. Bagshaw for having kindly proofread the manuscript.

\section{REFERENCES}

[1] A. Croisier, D. Esteban, and C. Galand, "Perfect channel splitting by use of interpolation/decimation/tree decomposition techniques," in Proc. Int. Conf. Inform. Sci. Syst., Patras, Greece, Aug. 1976, pp. 443-446.
[2] J. D. Johnston, "A filter family designed for use in quadrature mirror filter banks," in Proc. IEEE Int. Conf. Acoust., Speech, Signal Process., Apr. 1980, pp. 291-294.

[3] F. Mintzer, "Filters for distortion-free two-band multirate filter banks," IEEE Trans. Acoust., Speech, Signal Processing, vol. ASSP-33, June 1985

[4] M. J. T. Smith and T. P. Barnwell, "Exact reconstruction techniques for tree-structured subband coders," IEEE Trans. Acoust., Speech, Signal Processing, vol. ASSP-34, pp. 434-441, June 1986.

[5] M. Vetterli, "A theory of multirate filter banks," IEEE Trans. Acoust., Speech, Signal Processing, vol. ASSP-35, pp. 356-372, May 1987.

[6] P. P. Vaidyanathan, "Multirate digital filters, filter banks, polyphase networks, and applications: A tutorial," Proc. IEEE, vol. 78, pp. 56-93, Jan. 1990.

[7] P. P. Vaidyanathan, T. Q. Nguyen, Z. Doğanata, and T. Saramäki, "Improved technique for design of perfect reconstruction FIR QMF banks with lossless polyphase matrices," IEEE Trans. Acoust., Speech, Signal Processing, vol. 37, pp. 1042-1056, July 1989.

[8] M. Vetterli and D. Le Gall, "Perfect reconstruction filter banks: Some properties and factorizations," IEEE Trans. Acoust., Speech, Signal Processing, vol. 37, pp. 1057-1071, July 1989.

[9] T. Q. Nguyen and P. P. Vaidyanathan, "Structures for $M$-channel perfect-reconstruction FIR QMF banks which yield linear-phase analysis filters," IEEE Trans. Signal Processing, vol. 38, pp. 433-446, Mar. 1990.

[10] S. Mallat, "A theory for multiresolution signal decomposition: The wavelet decomposition," IEEE Trans. Pattern Anal. Machine Intell., vol. 11, pp. 674-693, July 1989.

[11] Y. Meyer, Ondelettes. Paris, France: Hermann, 1990, in French.

[12] I. Daubechies, "Orthonormal bases of compactly supported wavelets," Commun. Pure Appl. Math., vol. XLI, pp. 909-996, Nov. 1988.

[13] P. P. Vaidyanathan and P. Q. Hoang, "Lattice structures for optimal design and robust implementation of two-channel perfect reconstruction QMF banks," IEEE Trans. Acoust., Speech, Signal Processing, vol. 36, pp. 81-94, Jan. 1988.

[14] O. Rioul and P. Duhamel, "A remez exchange algorithm for orthonormal wavelets," IEEE Trans. Circuits Syst., vol. 41, pp. 550-560, Aug. 1994.

[15] K. Nayebi, T. P. Barnwell, III, and M. J. T. Smith, "The design of perfect reconstruction nonuniform band filter banks," in Proc. IEEE Int. Conf. Acoust., Speech, Signal Process., Toronto, Ont., Canada, May 1991, vol. 3, pp. 1781-1784.

[16] J. Kovačević and M. Vetterli, "Perfect reconstruction filter banks with rational sampling factors," IEEE Trans. Signal Processing, vol. 41, pp. 2047-2066, June 1993

[17] T. Blu, "Iterated filter banks with rational rate changes-Connection with discrete wavelet transforms," IEEE Trans. Signal Processing, vol. 41, pp. 3232-3244, Dec. 1993.

[18] — "Bancs de filtres itérés en fraction d'octave-Application au codage de son," Ph.D. Dissertation, École Nat. Supér. Télécommun., Paris, France, 1996, in French.

[19] J. Princen, "The design of nonuniform modulated filterbanks," IEEE Trans. Signal Processing, vol. 43, pp. 2550-2560, Nov. 1995.

[20] S. Wada, "Design of nonuniform division multirate fir filter banks," IEEE Trans. Circuits Syst. II, vol. 42, pp. 115-121, Feb. 1995.

[21] T. Blu, "Lossless filter design in two-band rational filter banks: A new algorithm," in Proc. GRETSI, Juan-les-Pins, France, Sept. 1993, vol. 1, pp. 69-72.

[22] J. Kovačević, "Filter banks and wavelets: Extensions and applications," Ph.D. dissertation, Columbia Univ., New York, NY, 1991.

[23] P. Q. Hoang and P. P. Vaidyanathan, "Non-uniform multirate filter banks: Theory and design," in Proc. IEEE Int. Symp. Circuits Syst., Portland, OR, 1989, pp. 371-374.

[24] T. Blu, "An iterated rational filter bank for audio coding," in Proc. TFTS, Paris, France, June 1996, pp. 81-84.

[25] A. Cohen and I. Daubechies, "Orthonormal bases of compactly supported wavelets III. Better frequency resolution," SIAM J. Math. Anal., vol. 24, no. 2, pp. 520-527, Mar. 1993.

[26] I. Daubechies and J. Lagarias, "Two-scale difference equations I. Existence and global regularity of solutions," SIAM J. Math. Anal., vol. 22, no. 5, pp. 1388-1410, Sept. 1991.

[27] O. Rioul, "Simple regularity criteria for subdivision schemes," SIAM J. Math. Anal., vol. 23, no. 6, pp. 189-194, Nov. 1992

[28] O. Rioul and T. Blu, "Simple regularity criteria for subdivision schemes II. The rational case," submitted for publication.

[29] Z. Cvetkovič and M. Vetterli, "Oversampled filter banks," IEEE Trans. Signal Processing, vol. 46, pp. 1245-1255, May 1998.

[30] Z. Doganata, P. P. Vaidyanathan, and T. Q. Nguyen, "General synthesis procedures for fir lossless transfer matrices, for perfect-reconstruction multirate filter bank applications," IEEE Trans. Signal Processing, vol. 36, pp. $1561-1574$, Oct. 1988 
[31] O. Rioul, "Ondelettes régulières: Application à la compression d'images fixes,” Ph.D. dissertation, École Nat. Supér. Télécommun., Paris, France, 1993, in French.

[32] K. Nayebi, T. P. Barnwell, III, and M. J. T. Smith, "Time-domain filter bank analysis: A new design theory," IEEE Trans. Signal Processing, vol. 40, pp. 1412-1429, June 1992.

[33] E. Zwicker and H. Fastl, Psychoacoustics. New York: Springer, 1990.

[34] T. Blu, "Shift error in iterated rational filter banks," in Proc. EUSIPCO, Trieste, Italy, Sept. 1996, vol. II, pp. 1199-1202.

[35] O. Rioul, "On the choice of wavelet filters for still image compression," in Proc. IEEE Int. Conf. Acoust., Speech, Signal Process., Minneapolis, MN, Apr. 1993, vol. V, pp. 550-553.

[36] R. E. Crochiere and L. R. Rabiner, "Interpolation and decimation of digital signals-A tutorial review," in Proc. IEEE, vol. 69, pp. 300-331, Mar. 1991.

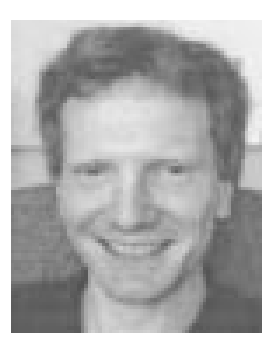

Thierry Blu was born in Orléans, France, in 1964. He graduated from École Polytechnique, Paris, France, in 1986 and from Télécom Paris (ENST), France, in 1988. In 1996, he received the Ph.D. degree in electrical engineering from ENST for a study on iterated rational filter banks applied to wide band audio coding.

$\mathrm{He}$ is currently with the Swiss Federal Institute of Technology, Lausanne, Switzerland, on leave from France Télécom-CNET (National Center for Telecommunications Studies), Issy-les-Moulineaux, France. His research interests include (multi-)wavelets, multiresolution analysis, multirate filter banks, approximation and sampling theory, and psychoacoustics. 